\title{
UNSTEADY MASS LOSS IN CLOSE BINARIES
}

\author{
R. F. WE B B I N K \\ Institute of Astronomy, Madingley Road, Cambridge CB3 OHA, England
}

\begin{abstract}
The evolution of a system of $1.50 M_{\odot}$ and $0.50 M_{\odot}$ components with initial separation $3.00 R_{\odot}$ is presented. The primary reaches its Roche lobe after $6.108 \times 10^{8} \mathrm{yr}$, and the mass loss rate grows quickly to $\sim 1.1 \times 10^{-7} M_{\odot} \mathrm{yr}^{-1}$. The deep convective envelope of the secondary contracts in response to accretion, however, and the system avoids contact, even though the main sequence mass-radius relationship crosses the Roche relationship at $0.758 M_{\odot}$.

The growth of the convective envelope of the primary with mass loss prevents thermaltimescale mass transfer from reaching any stable value. The mass loss rate continues to increase as a result of the tendency of the growing convective envelope to expand with mass loss. A Bath-type pulsational instability in the mass transfer rate is manifested as a longer-period thermal instability in the hydrostatic treatment. Eventually mass loss proceeds on a dynamical timescale, reaching $1.5 \times 10^{-4} M_{\odot} \mathrm{yr}^{-1}$. This is followed by a detached phase with the mass ratio not yet reversed, and a second dynamical episode leaving a $0.6666 M_{\odot}+1.3334 M_{\odot}$ binary still in a semi-detached state. The original secondary, now more massive, has a surface luminosity of $0.0538 L_{\odot}$ but a nuclear luminosity of $1540 L_{\odot}$, and a convective core of $1.076 M_{\odot}$ separated by a radiative zone of only $0.028 M_{\odot}$ from its convective envelope.

An attempt is made to follow the evolution of the secondary in the case where the stream energy, much greater than the intrinsic luminosity of the secondary, is dissipated spherically symmetrically at its surface. The outer envelope departs radically from the previous case, an optically deep radiative surface layer forming, and the convection zone splitting into two, the lower one remaining very massive. Because the mass transfer timescale is short compared with the thermal timescale of the secondary, however, it is concluded that the stream luminosity cannot significantly alter the evolution of the secondary.
\end{abstract}

